\title{
Preparation and evaluation of cyclodextrin polypseudorotaxane with PEGylated liposome as a sustained release drug carrier
}

\author{
Kayoko Hayashida ${ }^{1}$, Taishi Higashi ${ }^{1}$, Daichi Kono ${ }^{1}$, Keiichi Motoyama ${ }^{1}$, Koki Wada ${ }^{2}$ \\ and Hidetoshi Arima*1,3
}

\section{Full Research Paper \\ Address: \\ ${ }^{1}$ Graduate School of Pharmaceutical Sciences, Kumamoto University, 5-1 Oe-honmachi, Chuo-ku, Kumamoto 862-0973, Japan, ${ }^{2}$ Nihon Shokuhin Kako Co., Ltd., 30 Tajima, Fuji, Shizuoka 417-8539, Japan and ${ }^{3}$ Program for Leading Graduate Schools "HIGO (Health life science: Interdisciplinary and Glocal Oriented) Program", Kumamoto University, 5-1 Oe-honmachi, Chuo-ku, Kumamoto 862-0973, Japan \\ Email: \\ Hidetoshi Arima* - arimah@gpo.kumamoto-u.ac.jp \\ * Corresponding author}

\section{Keywords:}

cyclodextrins; doxorubicin; PEGylated liposome; polypseudorotaxane; sustained release

\author{
Beilstein J. Org. Chem. 2014, 10, 2756-2764. \\ doi:10.3762/bjoc. 10.292
}

Received: 13 July 2014

Accepted: 05 November 2014

Published: 25 November 2014

This article is part of the Thematic Series "Superstructures with cyclodextrins: Chemistry and applications II".

Guest Editor: G. Wenz

(C) 2014 Hayashida et al; licensee Beilstein-Institut.

License and terms: see end of document.

\begin{abstract}
Cyclodextrins (CDs) can form polypseudorotaxanes (PPRXs) with drugs or drug carriers possessing linear polymers such as polyethylene glycol (PEG). On the other hand, PEGylated liposomes have been utilized as a representative anticancer drug carrier. However, little is known about the formation of CD PPRX with PEGylated liposome. In the present study, we first report the formation of CD PPRX with PEGylated liposome and evaluate it as a sustained release drug carrier. PEGylated liposome encapsulating doxorubicin was disrupted by the addition of $\alpha$-CD. Meanwhile, $\gamma$-CD included two PEG chains and/or one bending PEG chain of PEGylated liposome and formed PPRX without the disruption of the membrane integrity of the PEGylated liposome. Moreover, the release of doxorubicin and/or PEGylated liposome encapsulating doxorubicin from the PPRX was prolonged in accordance with the matrix type release mechanism. These findings suggest the potential of $\gamma$-CD PPRX as sustained release carriers for PEGylated liposome products.
\end{abstract}

\section{Introduction}

Cyclodextrins (CDs) are cyclic oligosaccharides comprising six $(\alpha-C D)$, seven $(\beta-C D)$, and eight $(\gamma-C D)$ glucopyranose units. They are characterized by a hydrophobic central cavity and a hydrophilic outer surface [1,2]. CDs are acknowledged to form inclusion complexes with various hydrophobic drugs, and improve their pharmaceutical properties [3]. For instance, $\gamma$-CD 
forms inclusion complexes with doxorubicin (DOX) with a stability constant of $345 \mathrm{M}^{-1}$ [4]. CDs interact with cholesterol, phospholipids and proteins of biological membranes in the higher concentration range. Thus, CDs are utilized for studying the functions of caveolae, lipid rafts, and cholesterol transporters in various fields of cell biology [5]. Interestingly, CDs can also form inclusion complexes with linear polymers. Harada et al. have reported that a number of $\alpha$-CDs spontaneously thread onto polyethylene glycol (PEG) and form necklace-like supramolecular assemblies [6,7]. The latter are referred to as polypseudorotaxanes (PPRXs), since the release of $\alpha$-CD from the polymer chain can be achieved upon dissolution in water. The assembly of PPRX complexes is a sizedependent process, whereby the small cavity of $\alpha$-CD assembles with PEG, while the large cavity of $\beta$-CD forms the PPRX with polypropylene glycol (PPG) [6-8]. In addition, $\gamma$-CD forms PPRX with double strand PEG chains [9]. In this case, $\gamma$-CD not only includes two extended PEG chains but also one bent PEG chain. Actually, our research group and Gao et al. have reported that $\gamma$-CD can form PPRXs with bulky molecules-appended PEG derivatives, implying the formation of $\gamma$-CD PPRX with one bent PEG chain $[10,11]$. On the other hand, the covalent capping of both ends of the polymer chains in PPRXs with bulky molecules results in the trapping of CDs, which in this case cannot be de-threaded from the assembly, hence giving rise to polyrotaxanes $[12,13]$.

Recently, PPRXs and polyrotaxanes have been utilized as drug carriers for low-molecular weight drugs [14,15], protein drugs $[16,17]$, and nucleic acids [18-20]. We have also developed a number of PPRXs with various drugs or drug carriers and utilized them as controlled release systems. For example, $\gamma-\mathrm{CD}$ formed PPRX with coenzyme Q10, improving the solubility and bioavailability of coenzyme Q10 [21,22]. Also, $\alpha$ - and $\gamma$-CDs formed PPRXs with PEGylated proteins and provided sustained release profiles of PEGylated insulin and PEGylated lysozyme in vitro and in vivo [10,23-25]. Furthermore, $\alpha$ - and $\gamma$-CDs PPRXs with PEGylated PAMAM dendrimer and $\alpha$-CDappended PEGylated PAMAM dendrimer were useful as sustained gene transfer carriers [26,27].

Liposomes (LPs) are microscopic phospholipid vesicles with a bilayered membrane structure and are used as a promising drug carrier [28]. When conventional LPs are administrated intravenously, they are coated with plasma proteins, which results in a rapid removal from the systemic circulation by the reticuloendothelial system (RES). To produce long-circulating LPs, hydrophilic polymers, carbohydrates, peptides and proteins have been used to modify the surface of LPs [29]. Additionally, the targeting efficiency of LPs has been improved by appending various targeting-ligands such as antibodies, sugars and folic acid to LPs [30-32]. Recently, stimulus responsive LPs such as bubble LPs, $\mathrm{pH}$ responsive LPs and thermoresponsive LPs have been developed as smart drug carriers [33-35].

PEGylated LP (PEG-LP) is one of the most popular LP products and forms a hydrophilic layer on the surface of LPs [28]. It is not recognized by RES, which leads to a prolonged retention in circulation (stealth characteristics), and shows the enhanced permeability retention (EPR) effect [36]. PEG-LP is widely used as a drug carrier to realize the targeted drug delivery of anticancer drugs [28]. PEG-LP encapsulating DOX is commercially available as DOXIL/CAELYX ${ }^{\circledR}$ [28]. In addition, PEGLPs are also utilized as long-circulating drug carriers for protein drugs and nucleic acids [37,38]. Thus, PEG-LPs are representative drug carriers and CD PPRXs of PEG-LPs could be promising long-acting drug carriers. However, little is known about the formation of PPRXs with CDs.

CDs are known to disrupt LP due to their interaction with membrane components such as phospholipids and/or cholesterol at higher concentration $[39,40]$, even though CD PPRXs are generally prepared with $\mathrm{CD}$ solutions at high concentration. Therefore, it should be taken particular care to prepare CD PPRXs of PEG-LP. In the present study, we report on the first preparation and evaluation of CD PPRXs with PEG-LP as a sustained release drug carrier.

\section{Results and Discussion Interaction of phospholipid with CDs}

CDs disrupt LP by their interaction with lipid membranes. In particular, $\alpha-C D$ strongly interacts with phospholipids $[5,39,40]$. On the other hand, to prepare CD PPRXs, PEG should react with CDs in aqueous solution at high concentration of CDs. Thus, we should take particular care to prepare CD PPRXs of PEG-LP. First, to examine the interaction between CDs and phospholipids, both compounds were mixed in phosphate-buffered saline (PBS). As shown in Figure 1A, $\alpha$ - and $\gamma$-CDs formed precipitates with hydrogenated soybean phosphatidylcholine (HSPC) after mixing in PBS. Then, the powder $\mathrm{X}$-ray diffraction was measured after collecting the precipitates (Figure 1B). Both precipitates showed the channel-type crystal patterns [41] which indicate the interaction of $\alpha$ - and $\gamma$-CDs with HSPC.

Next, to investigate disruptive effects of CDs on LP encapsulating DOX (DOX/LP) or PEG-LP encapsulating DOX (DOX/ PEG-LP), DOX retained into DOX/LP or DOX/PEG-LP was quantitated $12 \mathrm{~h}$ after incubation with CDs (Figure 1C). After the incubation with $36.3-145 \mathrm{mg} / \mathrm{mL} \alpha$-CD solutions, approximately $70 \%$ and $60 \%$ of DOX was released from DOX/LP and DOX/PEG-LP, respectively, although the concentration 
(A)

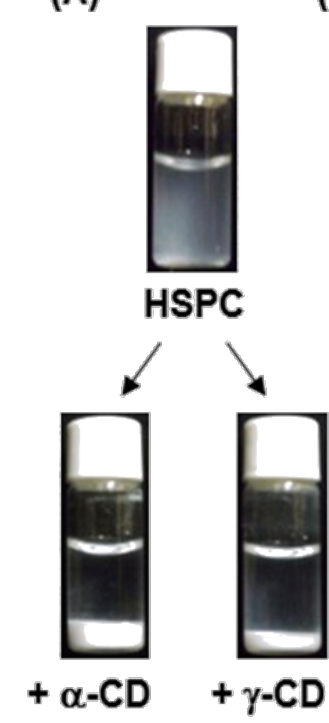

(B)

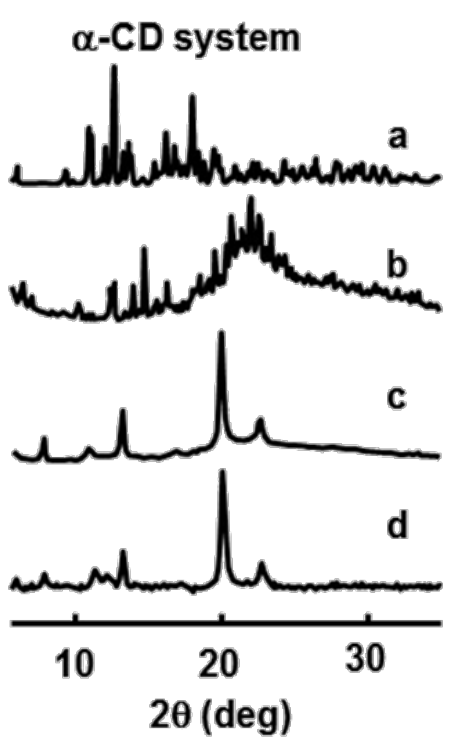

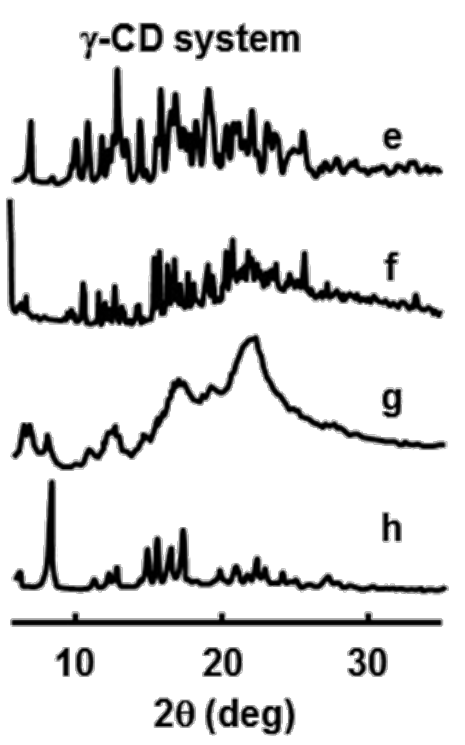

(C)

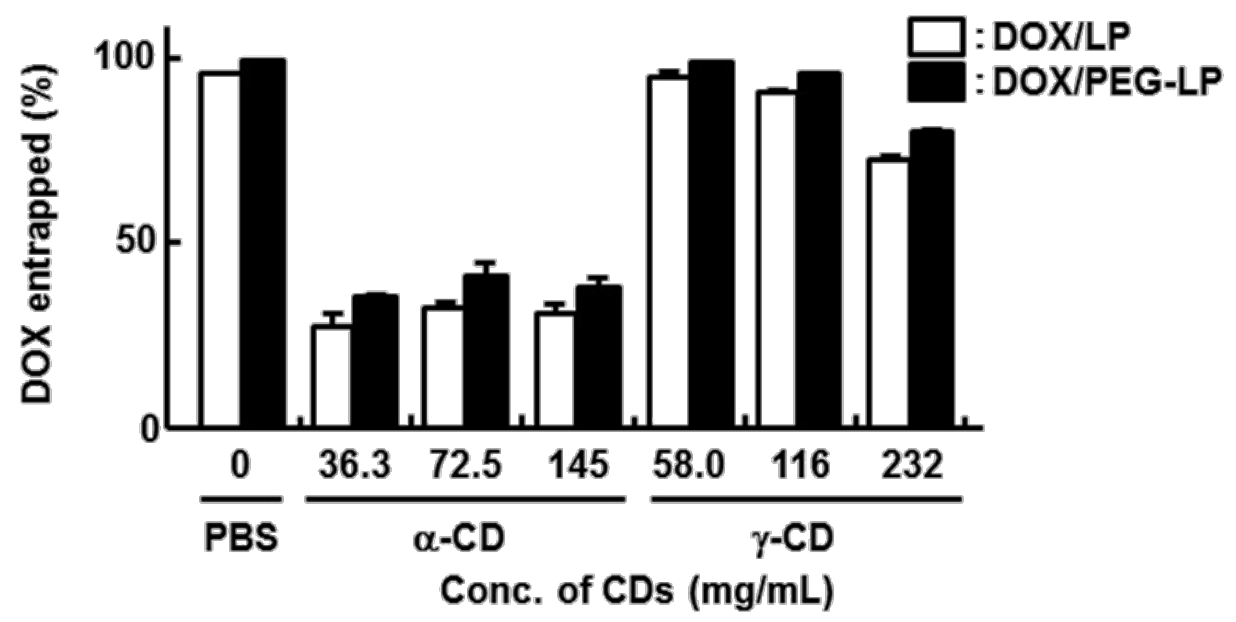

Figure 1: (A) Photographs and (B) powder X-ray diffraction patterns of precipitates formed by mixing the CD solutions and HSPC suspensions. (a) a-CD alone, (b) HSPC/a-CD physical mixture, (c) HSPC/a-CD complex, (d) PEG/a-CD PPRX, (e) $\gamma$-CD alone, (f) HSPC/ $\mathrm{Y}-\mathrm{CD}$ physical mixture, (g) HSPC/Y-CD complex, (h) PEG/Y-CD PPRX. (C) Effects of CDs on the entrapment ratio of DOX into LP or PEG-LP.

dependence of the DOX entrapment ratio was not observed in the $\alpha$-CD system. These concentrations of $\alpha-C D$ are high enough to completely disrupt the liposome membrane [42], which implies that the DOX entrapment ratio reaches the plateau at $36.3 \mathrm{mg} / \mathrm{mL} \alpha-\mathrm{CD}$. In addition, the DOX entrapment ratio in the $\alpha$-CD systems may be somewhat high. Conceivably, some DOXs may form aggregates in this experimental condition. These results, however, suggest the disruptive effects of $\alpha$-CDs on DOX/LP and DOX/PEG-LP, although additional evaluations are required. Meanwhile, in the case of the $\gamma-\mathrm{CD}$ system, more than $90 \%$ of DOX was retained into DOX/LP or DOX/PEG-LP after the incubation at 58 or $116 \mathrm{mg} / \mathrm{mL} \gamma-\mathrm{CD}$, although more than $20 \%$ of DOX was released from DOX/LP or DOX/PEG-LP in the presence of $232 \mathrm{mg} / \mathrm{mL} \gamma$-CD These results suggest that DOX/LP and DOX/PEG-LP are not disrupted by 58 or $116 \mathrm{mg} / \mathrm{mL} \gamma-\mathrm{CD}$, even though $36.3-145 \mathrm{mg} / \mathrm{mL} \alpha-\mathrm{CD}$ and $232 \mathrm{mg} / \mathrm{mL} \gamma-\mathrm{CD}$ show disruption effects on DOX/LP and DOX/PEG-LP. Hereafter, a fluorescence quenching experiment should be performed to obtain the additional evidences that LP structure is kept after the PPRX formation.

\section{Preparation of CD PPRXs with DOX/PEG-LP}

On the basis of the results of Figure 1, we prepared PPRX of DOX/PEG-LP with 58 and $116 \mathrm{mg} / \mathrm{mL} \gamma$-CD solution. Here, DOX/PEG-LP was prepared with HSPC/cholesterol/PEGylated 1,2-distearoyl-sn-glycero-3-phosphoethanolamine (PEG-DSPE, Figure 2A) (47:47:6, molar ratio). In addition, the particle size, polydispersity index (PDI), $\zeta$-potential and the drug entrapment ratio of DOX/PEG-LP were $132 \pm 1.53 \mathrm{~nm}, 0.07 \pm 0.00$, 
(A) DSPE

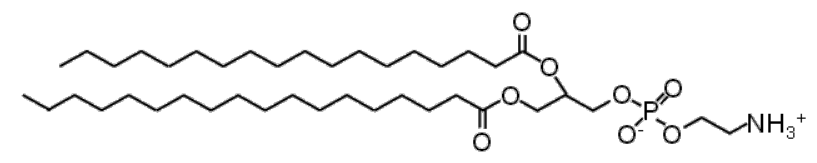

PEG-DSPE

(B)

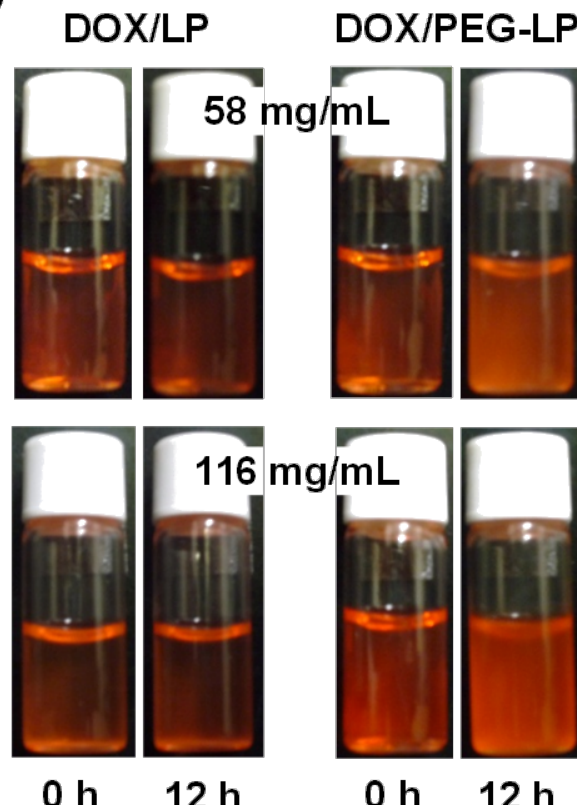

(C)

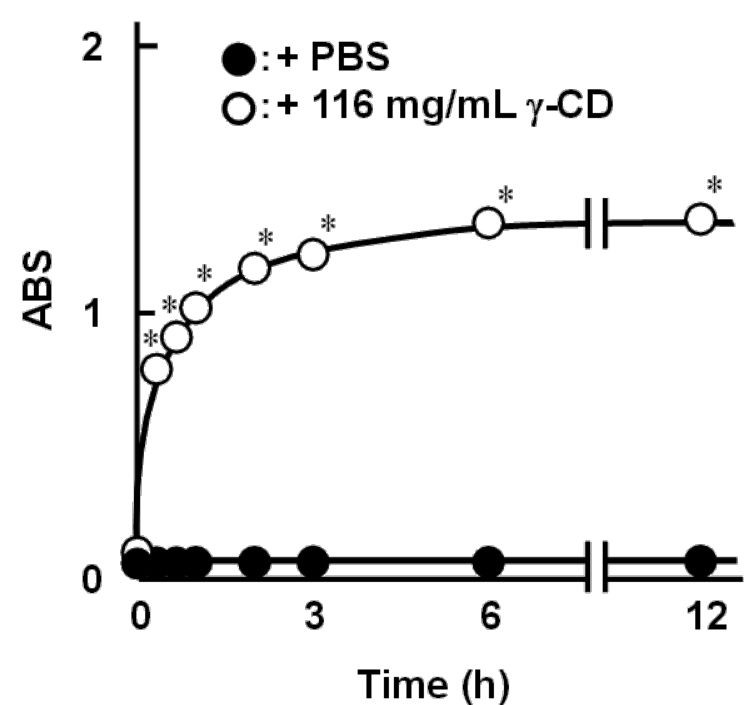

Figure 2: (A) Chemical structures of DSPE and PEG-DSPE, (B) photographs of the $y-C D$ solutions after adding DOX/LP or DOX/PEG-LP, and (C) turbidity of the DOX/PEG-LP solution in the absence and in the presence of $116 \mathrm{mg} / \mathrm{mL} \gamma-C D$. Each point represents the mean \pm S.E. of 3 experiments. ${ }^{*} p<0.05$ versus PBS.

$0.51 \pm 0.06$ and $99.6 \pm 0.07 \%$, respectively. When 58 and $116 \mathrm{mg} / \mathrm{mL} \gamma$-CD solutions were added to DOX/PEG-LP solution, precipitates were provided within $12 \mathrm{~h}$. Meanwhile, no precipitate was observed in the DOX/LP system, which suggests no formation of insoluble precipitate composed of $\gamma$-CD and phospholipids (Figure 2B). Moreover, the turbidity of the DOX/PEG-LP solution was increased in the presence of $116 \mathrm{mg} / \mathrm{mL} \gamma-\mathrm{CD}$, but not in the presence of PBS (Figure 2C). These results suggest that $\gamma$-CD forms PPRX with DOX/PEG-
$\mathrm{LP}$ at 58 or $116 \mathrm{mg} / \mathrm{mL}$ without a disruption of the membrane integrity of PEG-LP.

Table 1 shows approximate particle sizes, PDI and $\zeta$-potentials of the precipitates obtained by mixing of $\gamma$-CD and PEG-LP or DOX/PEG-LP solutions. In both systems, $\gamma$-CD formed microsized particles with PEG-LP or DOX/PEG-LP, exhibiting almost negligible $\zeta$-potentials, and an obvious difference was not observed. These results suggest that the presence of DOX in

Table 1: Particle sizes, PDI and $\zeta$-potentials of $Y$-CD PPRXs with PEG-LP and DOX/PEG-LP. ${ }^{a}$

\begin{tabular}{llll} 
System & Mean diameter $(\mathrm{nm})$ & PDI & $\zeta$-Potential $(\mathrm{mV})$ \\
\hline PEG-LP/Y-CD & $2543 \pm 112$ & $0.22 \pm 0.05$ & $-0.50 \pm 0.20$ \\
DOX/PEG-LP/Y-CD & $3097 \pm 317$ & $0.09 \pm 0.04$ & $0.28 \pm 0.29$ \\
\hline
\end{tabular}

aEach value represents the mean \pm S.E. of 3 experiments. 
PEG-LP scarcely alters the physicochemical properties of the PPRX. Additional studies by means of SEM or TEM may permit a more precise analysis and might confirm whether the LP structure is retained.

\section{Structure of CD PPRXs with PEG-LP}

To confirm whether the precipitate obtained in Figure 2 is CD PPRX with DOX/PEG-LP, we first examined its structure by using a FTIR spectrometer. A broad peak observed at $3,354 \mathrm{~cm}^{-1}$ of $\gamma$-CD in its physical mixture with DOX/PEG-LP was slightly shifted to $3,390 \mathrm{~cm}^{-1}$ in the DOX/PEG-LP/ $\gamma-C D$ system (Figure $3 \mathrm{~A}$ ). This shift is probably caused by the hydrogen bonds of the $\mathrm{O}-\mathrm{H}$ groups of $\gamma-\mathrm{CD}$ derived from PPRX formation [43].

There are three types of crystal packing of CD complexes, namely, the channel type, the cage type and the layer structure [44]. The powder X-ray diffractograms are useful for the confirmation of the PPRXs with CDs, as they provide enough information to distinguish between the herringbone packing of free CDs and the channel packing of inclusion complexes [13]. Therefore, the crystal structure of DOX/PEG-LP/ $\gamma-C D$ was determined by powder X-ray diffraction (Figure $3 \mathrm{~B}$ ). The diffraction peaks were observed at $2 \theta=7.43^{\circ}, 14.16^{\circ}, 16.65^{\circ}$ and $21.87^{\circ}$ in the DOX/PEG-LP/ $\gamma-C D$ system. Also, this diffraction pattern was different from that of the physical mixture of DOX/PEG-LP/ $\gamma$-CD and was almost the same as PEG/ $\gamma$ CD PPRX used as positive control (Figure 1B, h).

Moreover, the diffraction pattern of DOX/PEG-LP $/ \gamma-C D$ resembled the pattern of tetragonal columnar channels of the linearly aligned $\gamma$-CD cavities in the crystalline phase $[11,36]$. Therefore, the diffraction pattern of the DOX/PEG-LP/ $\gamma-C D$ was indexed on the basis of the two-dimensional tetragonal unit cells with dimensions $a=b=23.76 \AA$ as shown in Table 2. The $d$-spacing value of the $h k l$ (200) reflection was used to calculate the unit cell dimension. The calculated $d$-spacing $\left(d_{\text {cal }}\right)$ values were in excellent agreement with those observed $\left(d_{\text {obs }}\right)$ suggesting that DOX/PEG-LP/ $\gamma$-CD forms the tetragonal

Table 2: Crystallographic characteristics of $\mathrm{Y}-\mathrm{CD}$ PPRX with DOX/ PEG-LP.

\begin{tabular}{llll}
$2 \theta($ deg $)$ & $(h k l)$ & $d_{\text {obs }}(\AA)$ & $d_{\text {cal }}{ }^{\mathrm{a}}(\AA)$ \\
\hline 7.43 & $(200)$ & 11.88 & 11.88 \\
14.16 & $(400)$ & 5.93 & 5.94 \\
16.65 & $(420)$ & 5.32 & 5.31 \\
21.87 & $(530)$ & 4.06 & 4.07 \\
\hline
\end{tabular}

${ }^{\mathrm{a} C}$ Calculated assuming a tetragonal unit cell with $a=b=23.76 \AA$.

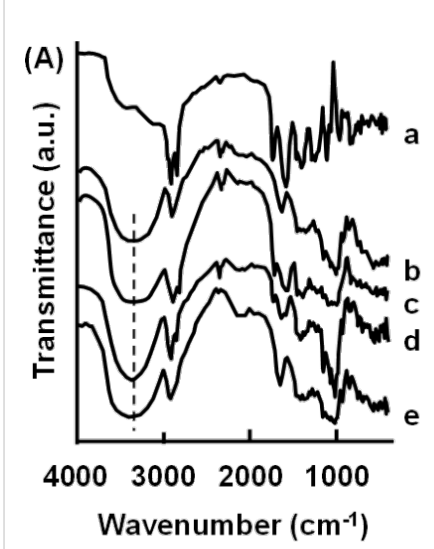

(B)

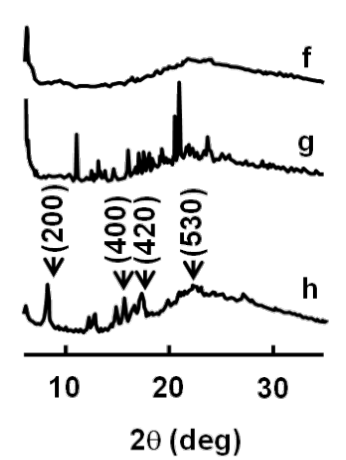

(C)

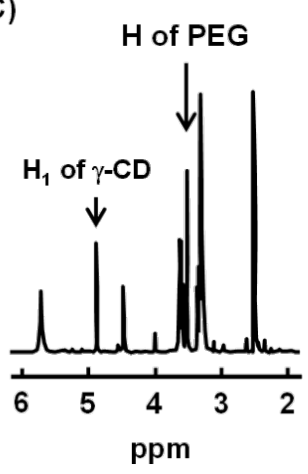

(D)

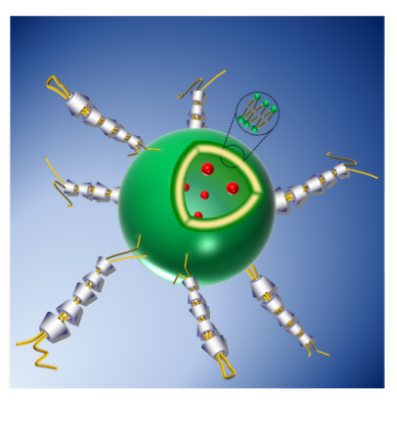

Figure 3: (A) FTIR spectra, (B) powder $X$-ray diffraction patterns, (C) ${ }^{1} \mathrm{H}$ NMR spectrum and (D) proposed partial structure of DOX/PEGLP/Y-CD PPRX. (a), (f) DOX/PEG-LP, (b) y-CD alone, (c), (g) DOX/ PEG-LP/Y-CD physical mixture, (d), (h) DOX/PEG-LP/Y-CD PPRX, (e) $P E G / Y-C D$ PPRX.

columnar structure. The results obtained by powder X-ray diffraction patterns suggest that $\gamma$-CD includes the PEG chain of DOX/PEG-LP and forms PPRX with tetragonal columnar structure. Additional experiments and analyses are required to determine the tetragonal columnar structure of DOX/PEG-LP/ $\gamma-\mathrm{CD}$ PPRX.

Next, to confirm the stoichiometry of the $\gamma$-CD PPRX with DOX/PEG-LP, a ${ }^{1} \mathrm{H}$ NMR spectrum was measured (Figure 3C). Approximately $12 \mathrm{~mol}$ of $\gamma$-CD were involved in the PPRX formation with one PEG chain in the DOX/PEG-LP, which indicates that four ethylene glycol repeating units are included in one $\gamma$-CD cavity [9]. In the case of $\alpha-C D$, two ethylene glycol repeating units of PEG are included in one $\alpha-C D$ cavity resulting in the formation of PPRX with one PEG chain [6,7]. Therefore, $\gamma$-CD likely forms PPRX with two PEG chains and/ or one bending PEG chain in DOX/PEG-LP.

Figure 3D shows the proposed schematic structure of $\gamma-C D$ PPRX with DOX/PEG-LP taking into consideration the results of Figure 3. $\gamma$-CD includes two PEG chains and/or one bending 
PEG chain of DOX/PEG-LP and forms PPRX. The PPRX moieties pack with a tetragonal columnar structure.

\section{Release profile of DOX or DOX/PEG-LP from the CD PPRX}

To evaluate the applicability of $\gamma$-CD PPRX with DOX/PEG-LP as a sustained release carrier for DOX, the release profile of DOX or DOX/PEG-LP from the CD PPRX was examined. Firstly, the total amount of DOX including naked DOX and encapsulated DOX in PEG-LP was quantitatively detected by fluorescence spectrometry. As shown in Figure 4A DOX/PEGLP was rapidly dissolved in PBS. On the other hand, the release of naked DOX or DOX/PEG-LP from $\gamma$-CD PPRX was prolonged and was accelerated by the increase of the volume of the dissolution medium (the rate: $3 \mathrm{~mL}>2 \mathrm{~mL}>1 \mathrm{~mL}$ ). Next, naked DOX and encapsulated DOX in PEG-LP were separately detected, and their release profiles were investigated (Figure $4 \mathrm{~B}$ ). Approximately $10-20 \%$ and $30-40 \%$ of DOX were released as naked DOX and encapsulated DOX in PEG$\mathrm{LP}$, respectively, in the $2 \mathrm{~mL}$ of dissolution medium system (Figure 4B). These results suggest that $\gamma$-CD PPRX with DOX/ PEG-LP shows the sustained release profile of DOX.

To investigate the release mechanism of naked DOX or DOX/ PEG-LP from $\gamma$-CD PPRX, the release kinetic profiles were applied to kinetic models such as zero order, first order, Higuchi and Hixson-Crowell equations [45]. The drug-release data obtained within $12 \mathrm{~h}$ in $1 \mathrm{~mL}$ of dissolution medium system were used. The release rate constants were calculated from the slope of the appropriate plots, and the correlation coefficient $(r)$ was also calculated (Table 3 ). The release kinetics of naked DOX or DOX/PEG-LP from $\gamma$-CD PPRX was well-fitted to the Higuchi's equation with a high $r$ value of 0.90 , indicating that the release shows the matrix type release profile.

In the matrix type release profile, the bases are either hydrophilic or hydrophobic. If a base is hydrophilic, the erosion and the dissolution of a base are observed among the drug

\begin{tabular}{|c|c|c|}
\hline Release model & Parameter & Value $^{a}$ \\
\hline Zero order & $\begin{array}{l}k_{0}(\% / h) \\
r\end{array}$ & $\begin{array}{l}1.72 \pm 0.06 \\
0.81 \pm 0.03\end{array}$ \\
\hline First order & $\begin{array}{l}k_{1}\left(\mathrm{~h}^{-1}\right) \\
r\end{array}$ & $\begin{array}{l}0.03 \pm 0.00 \\
0.84 \pm 0.03\end{array}$ \\
\hline Higuchi & ${ }_{r} k_{\mathrm{H}}\left(\% / \mathrm{h}^{1 / 2}\right)$ & $\begin{array}{l}7.31 \pm 0.26 \\
0.90 \pm 0.01\end{array}$ \\
\hline Hixson-Crowell & $k_{\mathrm{s}}(\% 1 / 3 / \mathrm{h})$ & $\begin{array}{l}0.03 \pm 0.01 \\
0.83 \pm 0.03\end{array}$ \\
\hline
\end{tabular}

aEach value represents the mean \pm S.E. of 3 experiments. (A) O: DOX/PEG-LP in $2 \mathrm{~mL}$ of PBS
$\gamma$-CD PPRX

$\triangle$ : in $3 \mathrm{~mL}$ of PBS

$\checkmark$ : in $2 \mathrm{~mL}$ of PBS

a : in $1 \mathrm{~mL}$ of PBS
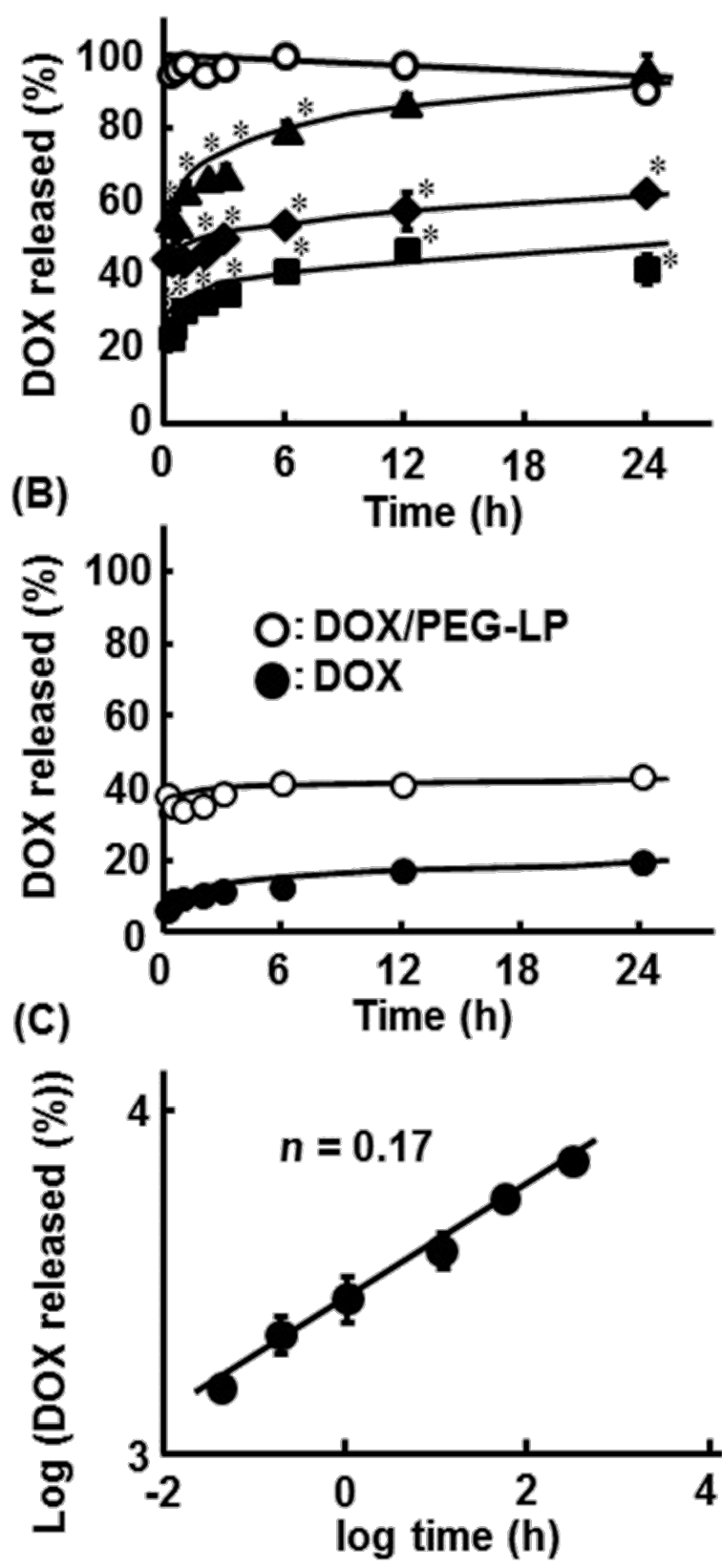

Figure 4: $(A)$ In vitro release profiles of the total DOX from $y$-CD PPRX in various volumes of $\mathrm{PBS},(\mathrm{B})$ release profiles of each naked DOX and DOX/PEG-LP in $2 \mathrm{~mL}$ of PBS, and (C) Korsmeyer-Peppas release profile of the total DOX from $Y$-CD PPRX. The amount of DOX was $0.2 \mathrm{mg}$ to $1 \mathrm{mg}$ of lipid. Each point represents the mean \pm S.E. of 3 experiments. ${ }^{*} p<0.05$ versus DOX/PEG-LP in $2 \mathrm{~mL}$ of PBS. 
release, and the Korsmeyer-Peppas model is often used. Therefore, we analyzed the release profile of naked DOX or DOX/ PEG-LP from $\gamma$-CD PPRX with the Korsmeyer-Peppas model which was developed to specifically model the release of a drug from a polymeric matrix. As shown in Figure 4C, naked DOX or DOX/PEG-LP released from $\gamma$-CyD PPRX showed a fair linearity, and the exponent value ( $n$ ) was 0.17 . In this model, the $n$ value characterizes the release mechanism of a drug. For instance, $n<0.5, n=0.5$ and $0.5<n \leq 1$ correspond to a quasiFickian, Fickian and non-Fickian diffusion mechanism, respectively [46]. Therefore, the release of naked DOX or DOX/PEGLP released from $\gamma$-CD PPRX is probably in accordance with a quasi-Fickian diffusion mechanism resulting from a coupling of the diffusion and erosion mechanism, and is controlled by more than one process [46]. Taken together, the proposed mechanism for the release of naked DOX or DOX/PEG-LP from $\gamma$-CD PPRX may be based on the dethreading of $\gamma$-CD from the PEG chain through the dilution, resulting in the erosion of $\gamma-C D$ PPRX and diffusion of the drug.

\section{Conclusion}

In the present study, we first investigated the formation of $\gamma$-CD PPRX with DOX/PEG-LP without disruption of the LP membrane integrity. $\gamma$-CD formed PPRX characterized by a tetragonal columnar structure with DOX/PEG-LP through the inclusion complexation with two PEG chains and/or one bending PEG chain of DOX/PEG-LP. Moreover, the resulting PPRX showed a sustained release profile of DOX and/or DOX/ PEG-LP. To clarify detailed structures of $\gamma$-CD PPRX with DOX/PEG-LP and its utility as a sustained release drug carrier, further studies are required. Especially, differences in the structures of $\gamma$-CD PPRX with DOX/PEG-LP could exert influence on the release rate of DOX/PEG-LP. It may be worthwhile to investigate the controlled release system of DOX/PEG-LP by means of the formation of PPRXs with various structures. Nevertheless, our findings provide useful information to design a novel sustained release system for PEG-LP products.

\section{Experimental Materials}

$\alpha$ - and $\gamma$-CDs were donated by Nihon Shokuhin Kako (Tokyo, Japan). DOX hydrochloride was purchased from Wako Pure Chemical Industries (Osaka, Japan). HSPC (COATSOME ${ }^{\mathrm{TM}}$ NC-21E), DSPE (COATSOME ${ }^{\mathrm{TM}}$ ME-8080) and PEG-DSPE (SUNBRIGHT $^{\mathrm{TM}}$ DSPE-020-CN, molecular weight of PEG = $2,000)$ were purchased from NOF corporation (Tokyo, Japan). Other chemicals and solvents were of analytical reagent grade.

\section{Interaction of CDs with HSPC}

HSPC (50 mg) was dissolved in $3 \mathrm{~mL}$ of chloroform. After evaporation and drying under reduced pressure overnight, $3 \mathrm{~mL}$ of water were added. The resulting suspension $(100 \mu \mathrm{L})$ was added to $900 \mu \mathrm{L}$ of $\alpha-\mathrm{CD}(145 \mathrm{mg} / \mathrm{mL})$ or $\gamma$-CD $(232 \mathrm{mg} / \mathrm{mL})$ aqueous solution, and the suspension was kept at $4{ }^{\circ} \mathrm{C}$ for $12 \mathrm{~h}$. After centrifugation $(12,000 \mathrm{rpm}, 10 \mathrm{~min})$, the supernatant was removed. The resulting precipitate was dried under reduced pressure, and then the powder X-ray diffraction was measured.

\section{Powder X-ray diffraction}

Powder X-ray diffraction patterns were measured by a Rigaku Ultima IV X-ray diffractometer (Tokyo, Japan) with a Ni filtered $\mathrm{Cu} \mathrm{K} \alpha$ radiation, a voltage of $40 \mathrm{kV}$, a current of $40 \mathrm{~mA}$, a scanning speed of $5 \% \mathrm{~min}$, a time constant of $2 \mathrm{~s}$, and a scan range of $2 \theta=5-35^{\circ}$.

\section{Effects of CDs on the entrapment ratio of DOX into LP or PEG-LP}

DOX/PEG-LP was prepared with HSPC/cholesterol/PEG-DSPE (47:47:6, molar ratio) according to the method previously reported by Arima et al. [47]. The DOX/PEG-LP solution $(20 \mu \mathrm{L})$ was added to $500 \mu \mathrm{L}$ of $\alpha-\mathrm{CD}(36.3,72.5$ and $145 \mathrm{mg} / \mathrm{mL})$ or $\gamma$-CD $(58.0,116$ and $232 \mathrm{mg} / \mathrm{mL})$ PBS solution, and the suspension was kept at $4{ }^{\circ} \mathrm{C}$ for $12 \mathrm{~h}$. After 20 -fold dilution with PBS and ultracentrifugation $(50,000 \mathrm{rpm}, 60 \mathrm{~min})$, the fluorescence intensity of the supernatant was measured by a F-4500 fluorescence spectrometer (Hitachi, Tokyo, Japan) at $\lambda_{\mathrm{em}}=554 \mathrm{~nm}\left(\lambda_{\mathrm{ex}}=470 \mathrm{~nm}\right)$.

\section{Preparation of CD PPRX with DOX/PEG-LP}

The DOX/PEG-LP solution $(40 \mu \mathrm{L})$ was added to $1000 \mu \mathrm{L}$ of $\gamma$-CD $(58.0$ or $116 \mathrm{mg} / \mathrm{mL})$ PBS solution, and the suspension was kept at $4{ }^{\circ} \mathrm{C}$ for $12 \mathrm{~h}$. The turbidity of the resulting suspension was measured with a JASCO V-630 UV-visible spectrophotometer (Tokyo, Japan) at $800 \mathrm{~nm}$. To obtain the solid sample of the PPRX, the supernatant was removed after the centrifugation (12,000 rpm, $10 \mathrm{~min})$. The resulting precipitate was dried under reduced pressure.

\section{Particle size, $P D I$ and $\zeta$-potential of $C D$ PPRX with DOX/PEG-LP}

The particle size, PDI and $\zeta$-potential of the suspension of $\gamma$-CD PPRX with DOX/PEG-LP were determined by dynamic light scattering by using a Zetasizer Nano (Malvern Instruments, Worcestershire, UK). The dynamic light scattering was analyzed by the general purpose mode. The measurements were carried out at least in triplicates.

\section{FTIR}

The sample preparation was performed by using the $\mathrm{KBr}$ method. The FTIR spectrum of $\gamma$-CD PPRX with DOX/PEG-LP was recorded on a JIR-6500W FTIR spectrometer (JEOL, 
Tokyo, Japan) in the range between 4,000 and $400 \mathrm{~cm}^{-1}$ with a resolution of $4 \mathrm{~cm}^{-1}$ and 16 scans.

\section{${ }^{1} \mathrm{H}$ NMR}

${ }^{1} \mathrm{H}$ NMR spectrum was taken at $25{ }^{\circ} \mathrm{C}$ on a JEOL $\alpha-500$ FT-NMR (Tokyo, Japan) operating at $500 \mathrm{MHz}$ by using a $5 \mathrm{~mm}$ sample tube. Deuterated DMSO (DMSO- $d_{6}$ ) was used as a solvent. The stoichiometry of the $\gamma$-CD PPRX with DOX/ PEG-LP was determined by measuring peak areas of the anomeric proton of CDs and the ethylene protons of the PEGLP.

\section{In vitro release study}

The various volumes (1.0, 2.0 or $3.0 \mathrm{~mL}$ ) of PBS (pH 7.4) were added to the suspension including $\gamma$-CD PPRX with DOX/PEG$\mathrm{LP}$ at $37^{\circ} \mathrm{C}$ and stirred at $100 \mathrm{rpm}$. At appropriate intervals, $100 \mu \mathrm{L}$ of the dissolution medium were withdrawn, centrifuged at 5,000 rpm for $5 \mathrm{~min}$, and 20-fold diluted with PBS. After the addition of Triton ${ }^{\mathrm{TM}} \mathrm{X}-100(10 \mu \mathrm{L})$, the samples were analyzed by a F-4500 fluorescence spectrometer (Hitachi, Tokyo, Japan) at $\lambda_{\mathrm{em}}=590 \mathrm{~nm}\left(\lambda_{\mathrm{ex}}=470 \mathrm{~nm}\right)$.

The release kinetics of DOX or DOX/PEG-LP from the $\gamma$-CD PPRX was evaluated according to zero order kinetics, first order kinetics, Higuchi's model, Hixson-Crowell model and Korsmeyer-Peppas's model.

Zero order kinetics

$$
\mathrm{Q}_{\mathrm{t}}=\mathrm{Q}_{0}-k_{0} t
$$

where $Q_{t}$ is the amount of drug remaining in solid state at time $t, \mathrm{Q}_{0}$ is the initial amount of drug in the $\gamma$-CD PPRX and $k_{0}$ is the zero order release rate constant.

First order kinetics

$$
\ln \mathrm{Q}_{\mathrm{t}}=\ln \mathrm{Q}_{0}-k_{1} t
$$

where $\mathrm{Q}_{t}$ is the amount of drug remaining in solid state at time $t, \mathrm{Q}_{0}$ is the initial amount of drug in the $\gamma$-CD PPRX and $k_{1}$ is the first order release rate constant.

Higuchi's model

$$
\mathrm{Q}_{\mathrm{t}}=k_{\mathrm{H}} t^{1 / 2}
$$

where $\mathrm{Q}_{\mathrm{t}}$ is the amount of drug released in time $t$ and $k_{\mathrm{H}}$ is the Higuchi's (release) rate constant.
Hixson-Crowell model

$$
\mathrm{Q}_{0}^{1 / 3}-\mathrm{Q}_{\mathrm{t}}^{1 / 3}=k_{\mathrm{s}} t
$$

where $\mathrm{Q}_{0}$ is the initial amount of drug in the $\gamma$-CD PPRX, $\mathrm{Q}_{\mathrm{t}}$ is the amount of drug remaining in solid state at time $t$ and $k_{\mathrm{S}}$ is the Hixson-Crowell (release) rate constant.

Korsmeyer-Peppas's model

$$
M_{\mathrm{t}} / M_{\infty}=k_{\mathrm{P}} t^{\mathrm{n}}
$$

where $M_{\mathrm{t}} / M_{\infty}$ is fraction of drug release at time $t, k_{\mathrm{P}}$ is the release rate constant, and $\mathrm{n}$ is the release exponent.

\section{Data analysis}

Data were given as the mean \pm S.E. The statistical significance of mean coefficients for the studies was performed by analysis of variance followed by Scheffe's test. $p$-Values for significance were set at 0.05 .

\section{Acknowledgements}

The authors thank Nihon Shokuhin Kako Co., Ltd. (Tokyo, Japan) for providing parent CDs. This study was funded by Nihon Shokuhin Kako Co., Ltd. (Tokyo, Japan).

\section{References}

1. Szejtli, J. Med. Res. Rev. 1994, 14, 353-386. doi:10.1002/med.2610140304

2. Arima, H.; Motoyama, K.; Higashi, T. Adv. Drug Delivery Rev. 2013, 65, 1204-1214. doi:10.1016/j.addr.2013.04.001

3. Uekama, K.; Hirayama, F.; Irie, T. Chem. Rev. 1998, 98, 2045-2076. doi:10.1021/cr970025p

4. Bekers, O.; Beijnen, J. H.; Otagiri, M.; Bult, A.; Underberg, W. J. M. J. Pharm. Biomed. Anal. 1990, 8, 671-674. doi:10.1016/0731-7085(90)80100-4

5. Irie, T.; Uekama, K. J. Pharm. Sci. 1997, 86, 147-162. doi:10.1021/js960213f

6. Harada, A.; Kamachi, M. Macromolecules 1990, 23, 2821-2823. doi:10.1021/ma00212a039

7. Harada, A.; Li, J.; Kamachi, M. Macromolecules 1993, 26, 5698-5703. doi:10.1021/ma00073a026

8. Harada, A.; Kamachi, M. J. Chem. Soc., Chem. Commun. 1990, 1322-1323. doi:10.1039/c39900001322

9. Harada, A.; Li, J.; Kamachi, M. Nature 1994, 370, 126-128. doi:10.1038/370126a0

10. Higashi, T.; Hirayama, F.; Yamashita, S.; Misumi, S.; Arima, H.; Uekama, K. Int. J. Pharm. 2009, 374, 26-32. doi:10.1016/j.ijpharm.2009.02.017

11. Gao, P.; Wang, J.; Ye, L.; Zhang, A.-y.; Feng, Z.-g. Macromol. Chem. Phys. 2011, 212, 2319-2327. doi:10.1002/macp.201100319

12. Harada, A.; Li, J.; Kamachi, M. Nature 1992, 356, 325-327. doi:10.1038/356325a0 
13. Wenz, G.; Han, B.-H.; Müller, A. Chem. Rev. 2006, 106, 782-817. doi:10.1021/cr970027+

14. Yu, S.; Zhang, Y.; Wang, X.; Zhen, X.; Zhang, Z.; Wu, W.; Jiang, X. Angew. Chem., Int. Ed. 2013, 125, 7413-7418. doi:10.1002/ange.201301397

15. Higashi, K.; Ideura, S.; Waraya, H.; Moribe, K.; Yamamoto, K. Cryst. Growth Des. 2009, 9, 4243-4246. doi:10.1021/cg900573w

16. Ma, D.; Zhang, L.-M.; Xie, X.; Liu, T.; Xie, M.-Q. J. Colloid Interface Sci. 2011, 359, 399-406. doi:10.1016/j.jcis.2011.04.032

17. Li, Q.; Xia, B.; Branham, M.; Ha, W.; Wu, H.; Peng, S.-L.; Ding, L.-S.; Li, B.-J.; Zhang, S. Carbohydr. Polym. 2011, 86, 120-126. doi:10.1016/j.carbpol.2011.04.017

18. Li, J.; Loh, X. J. Adv. Drug Delivery Rev. 2008, 60, 1000-1017. doi:10.1016/j.addr.2008.02.011

19. Dandekar, P.; Jain, R.; Keil, M.; Loretz, B.; Muijs, L.; Schneider, M.; Auerbach, D.; Jung, G.; Lehr, C.-M.; Wenz, G. J. Controlled Release 2012, 164, 387-393. doi:10.1016/j.jconrel.2012.06.040

20. Ooya, T.; Choi, H. S.; Yamashita, A.; Yui, N.; Sugaya, Y.; Kano, A.; Maruyama, A.; Akita, H.; Ito, R.; Kogure, K.; Harashima, H. J. Am. Chem. Soc. 2006, 128, 3852-3853. doi:10.1021/ja055868+

21. Nishimura, K.; Higashi, T.; Yoshimatsu, A.; Hirayama, F.; Uekama, K.; Arima, H. Chem. Pharm. Bull. 2008, 56, 701-706. doi:10.1248/cpb.56.701

22. Higashi, T.; Nishimura, K.; Yoshimatsu, A.; Ikeda, H.; Arima, K.; Motoyama, K.; Hirayama, F.; Uekama, K.; Arima, H. Chem. Pharm. Bull. 2009, 57, 965-970. doi:10.1248/cpb.57.965

23. Higashi, T.; Hirayama, F.; Arima, H.; Uekama, K. Bioorg. Med. Chem. Lett. 2007, 17, 1871-1874. doi:10.1016/j.bmcl.2007.01.029

24. Higashi, T.; Hirayama, F.; Misumi, S.; Arima, H.; Uekama, K. Biomaterials 2008, 29, 3866-3871. doi:10.1016/j.biomaterials.2008.06.019

25. Higashi, T.; Hirayama, F.; Misumi, S.; Motoyama, K.; Arima, H.; Uekama, K. Chem. Pharm. Bull. 2009, 57, 541-544. doi:10.1248/cpb.57.541

26. Motoyama, K.; Hayashida, K.; Arima, H. Chem. Pharm. Bull. 2011, 59, 476-479. doi:10.1248/cpb.59.476

27. Motoyama, K.; Hayashida, K.; Higashi, T.; Arima, H. Bioorg. Med. Chem. 2012, 20, 1425-1433. doi:10.1016/j.bmc.2011.12.060

28. Torchilin, V. P. Nat. Rev. Drug Discovery 2005, 4, 145-160. doi:10.1038/nrd1632

29. Rani, D. T. Int. Res. J. Pharm. 2013, 4, 6-12.

30. Koren, E.; Apte, A.; Jani, A.; Torchilin, V. P. J. Controlled Release 2012, 160, 264-273. doi:10.1016/j.jconrel.2011.12.002

31. Zhou, X.; Zhang, M.; Yung, B.; Li, H.; Zhou, C.; Lee, L. J.; Lee, R. J. Int. J. Nanomed. 2012, 7, 5465-5474. doi:10.2147/IJN.S33965

32. Yamada, A.; Taniguchi, Y.; Kawano, K.; Honda, T.; Hattori, Y.; Maitani, Y. Clin. Cancer Res. 2008, 14, 8161-8168. doi:10.1158/1078-0432.CCR-08-0159

33. Suzuki, R.; Takizawa, T.; Negishi, Y.; Utoguchi, N.; Maruyama, K. Int. J. Pharm. 2008, 354, 49-55. doi:10.1016/j.ijpharm.2007.10.034

34. Li, T.; Takeoka, S. Int. J. Nanomed. 2013, 8, 3855-3866. doi:10.2147/IJN.S47749

35. Chen, K.-J.; Liang, H.-F.; Chen, H.-L.; Wang, Y.; Cheng, P.-Y.; Liu, H.-L.; Xia, Y.; Sung, H.-W. ACS Nano 2013, 7, 438-446. doi:10.1021/nn304474j

36. Matsumura, Y.; Maeda, H. Cancer Res. 1986, 46, 6387-6392.

37. Kim, A.; Yun, M.-O.; Oh, Y.-K.; Ahn, W.-S.; Kim, C.-K. Int. J. Pharm. 1999, 180, 75-81. doi:10.1016/S0378-5173(98)00408-6
38. Hatakeyama, H.; Akita, H.; Harashima, H. Biol. Pharm. Bull. 2013, 36, 892-899. doi:10.1248/bpb.b13-00059

39. Miyajima, K.; Saito, H.; Nakagaki, M. J. Pharmacobio-Dyn. 1987, 10 , 123-128.

40. Puskás, I.; Csempesz, F. Colloids Surf., B 2007, 58, 218-224. doi:10.1016/j.colsurfb.2007.03.011

41. Takeo, K.; Kuge, T. Agric. Biol. Chem. 1969, 33, 1174-1180. doi:10.1271/bbb1961.33.1174

42. Nishijo, J.; Shiota, S.; Mazima, K.; Inoue, Y.; Mizuno, H.; Yoshida, J. Chem. Pharm. Bull. 2000, 48, 48-52. doi:10.1248/cpb.48.48

43. Lu, J.; Shin, I. D.; Nojima, S.; Tonelli, A. E. Polymer 2000, 41, 5871-5883. doi:10.1016/S0032-3861(99)00773-9

44. Uekama, K. Yakugaku Zasshi 1981, 101, 857-873.

45. Dash, S.; Murthy, P. N.; Nath, L.; Chowdhury, P. Acta Pol. Pharm. 2010, 67, 217-223.

46. Apparao, P.; Prabhakarreddy, J. V.; Raju, J.; Shashidher, B. Der. Pharma. Sinica 2011, 2, 176-192.

47. Arima, H.; Hagiwara, Y.; Hirayama, F.; Uekama, K. J. Drug Targeting 2006, 14, 225-232. doi:10.1080/10611860600711136

\section{License and Terms}

This is an Open Access article under the terms of the Creative Commons Attribution License (http://creativecommons.org/licenses/by/2.0), which permits unrestricted use, distribution, and reproduction in any medium, provided the original work is properly cited.

The license is subject to the Beilstein Journal of Organic Chemistry terms and conditions:

(http://www.beilstein-journals.org/bjoc)

The definitive version of this article is the electronic one which can be found at: doi:10.3762/bjoc. 10.292 